\title{
The primary photochemical reaction step of unsubstituted indolino-spiropyrans
}

\author{
N.P. Ernsting, B. Dick, and Th. Arthen-Engeland \\ Abteilung Laserphysik, Max-Planck-Institut für biophysikalische Chemie, \\ D-3400 Göttingen, Federal Republic of Germany
}

\begin{abstract}
The photochemical ring opening reaction of unsubstituted indolino-spiropyrans in $n$-pentane, to the corresponding merocyanines, has been followed by transient absorption spectroscopy. The temporal resolution was better than $0.4 \mathrm{ps}$. During the pump pulse, an ultrafast unstructured absorption covers the entire measurement range 380-680 nm. From it emerges the structured absorption spectrum of a first merocyanine isomer, with typical time constants in the range $0.9-1.4 \mathrm{ps}$. The transient merocyanine spectra are compared to the spectra which are obtained when the spiropyrans are irradiated at low temperature in an argon matrix. In conjunction with semiempirical calculations, the first merocyanine isomer is assigned a trans-trans-cis structure.
\end{abstract}

\section{INTRODUCTION}

Spiropyrans consist of an (extended) pyran moiety, and a second moiety which is held orthogonal by a common spiro-carbon atom. The $\pi$-electron systems of both constituent haives do not interact because of their orthogonal orientation, so that the absorption spectrum of the compound is essentially the superimposition of the two constituent chromophores. Here we consider only spiropyrans in which the second moiety consists of the indoline chromophore. The parent compound 1 is shown in Fig. 1; it is commonly abbreviated BIPS for $1^{\prime}, 3^{\prime}, 3^{\prime}$-trimethylspiro-[2H-1-benzopyran-2,2'-indoline]. Upon excitation in the near UV, the bond between the spiro carbon atom and the oxygen atom is broken, the methine chain rearranges, and the two parts of the molecule are allowed to rotate relative to each other (2 to $\underline{5}$ in Fig. 1). Now the $\pi$-electrons may conjugate across the entire structure ( which may be classed among the merocyanine dyes) resulting in intense absorption in the range 500-600 nm. However, in many cases the merocyanine form of the molecule is thermodynamically unstable, and it reverts eventualiy to the original, colorless spiro form.

This "photochromism" of spiropyrans was first reported and intensely studied by E. Fischer and coworkers (ref. 1-3). Potential applications for displays, optical filters and data storage led to a surge of investigations into spiropyran photochemistry (ref. 4 gives an excelient review up to 1969). It appeared that efficient photochromism requires a nitro substituent on the benzopyran ring for two reasons. First, the nitro group increases the quantum yield of photocoloration by introducing a triplet pathway; second, it stabilizes the zwitterionic merocyanine form of the molecule, so that the thermal back reaction is slowed down. However, the photochemical stability is adversely affected by nitro substitution. This problem may be alleviated by using the related spiro-oxazines, which give reasonable photocoloration yields and stability without nitro substitution (ref. 5).

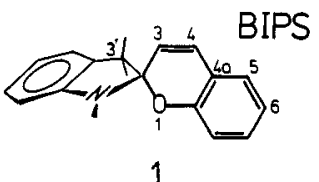

1

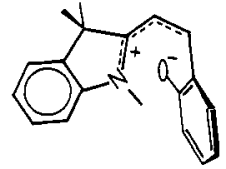

$\underline{4}$

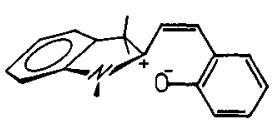

$\underline{2}$

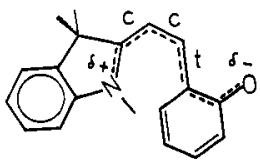

$\underline{5}$

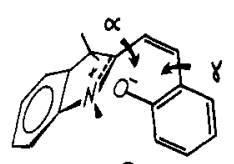

$\underline{3}$

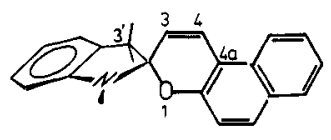

$\underline{6}$

Fig. 1. BIPS (1) , hypothetical structures for its first photochemical reaction product $X(\underline{2}-\underline{4})$, and for a merocyanine isomer $(\underline{5})$. 
The photochemistry of spiropyrans may conveniently be classed into the following areas:

Properties of the spiroform Geometry (ref.6); electronic structure, intramolecular energy transfer between the orthogonal parts, and dependence of quantum yields on excitation wavelength (ref. 7-10), and on medium and temperature (ref. 11, 12).

Merocyanine isomers Several near-planar isomers of the open form are conceivable, by rotation around the three central bonds $C_{2}-C_{3}, C_{3}-C_{4}$, and $C_{4}-C_{42}$. Evidence for these isomers was obtained when the photocoloration was carried out in rigid media at low temperature (ref.2, 3). A structured merocyanine absorption spectrum was thus generated, corresponding to a first isomer which was labelled $B_{1}$. On heating, the spectrum changed irreversibly to a similar one which may be associated with a second, more stable isomer $B_{2}$, and so on. The isomers interconvert thermally at room temperature, so that the kinetics of the thermal decoloration is determined by the isomer with the lowest activation energy for the back reaction. Several isomers have been characterized by resonance Raman spectroscopy (ref. 13-15). The structures of open isomeric forms have been reported (ref. 16).

\section{Dispersive kinetics of decoloration in solid media}

The thermal back reaction in solid media does not generally obey first-order kinetics. Instead the kinetics may be described as a superimposition of independent first-order processes, albeit with a broad distribution of rate constants. Each rate constant is associated with a certain free volume in the (e.g. polymer) matrix. Hence the distribution of rate constants reflects the glassy nature of the medium, rather than the intrinsic chemical reaction (ref. 17-19).

Aggregation of merocyanine isomers Irradiation of nitro-substituted spiropyrans in solution, and in an electrostatic field, leads to aggregates of the merocyanine form aligned in a "string-of-beads" structure. They precipitate in sub-micrometer sized globules which are composed of highly dipolar cores covered by amorphous envelopes (ref. 20).

\section{Primary photochemical reaction steps in color formation}

The participation of triplet states in the photocoloration of nitro-substituted spiropyrans has been shown by triplet sensitization ( ref. 4 and citations there; ref. 21) and by transient absorption measurements (ref. 22, 23). A triplet pathway is also responsible for aggregation of the resulting merocyanine isomers (ref. 22). By contrast, photocoloration of the unsubstituted parent compound BIPS and the spiro-naphthopyran 6 proceeds only through the singlet route (ref. 24$)$. Let us return for a moment to the distribution of merocyanine isomers. It has been suggested that the first isomer produced in the photochemical reaction should resemble the parent spiropyran geometry, i.e. the central $C_{3}-C_{4}$ bond should have a cisoid conformation as shown in structures 2 - 5 of Fig. 1. This primary cisoid isomer was labelled X (ref. 25). Ground and excited singlet and triplet states of $X$ have been implicated in the photocoloration of 6 nitro-BIPS (ref. 10, 22, 23, 26, 27).

Here we address the question whether a primary, cisoid merocyanine isomer exists, however briefly, on the picosecond time scale. We limit our investigation to the unsubstituted parent compounds BIPS (1) and the spiro-naphthopyran 6 , in order to avoid complications due to possible triplet states at early time. (For simplicity and somewhat loosely, we will call $\underline{6}$ naphtho-BIPS.)

We report

"MNDO calculations of the merocyanine isomers of BIPS in their ground state,

"transient absorption spectra of the spiropyran - merocyanine conversion, with 0.35 ps time resolution, and

"spectra of a merocyanine isomer isolated in an argon matrix at $10 \mathrm{~K}$.

\section{MNDO CALCULATIONS OF MEROCYANINE ISOMERS OF BIPS}

The AM1 modification of the MNDO-Hamiltonian has been used for all calculations. (Program QCPE 560). All geometry optimizations were performed on the SCF level with the PRECISE option, and all geometric parameters were optimized. Configuration interaction with all singly and doubly excited configuration between the two HOMOs and the two LUMOs (C.I.=4 option) yielded only a small energy correction. These latter calculations, which also gave vertical excitation energies, have been used to construct the energy level diagram in Fig. 3 .

The spiro form of BIPS is found at $\alpha=250^{\circ}, \quad \beta=0^{\circ}$, and $\gamma=-5^{\circ}$. This means that the second and third angle (which are located in the pyran ring) have cis configuration, whereas the first angle is such that the two molecular halves are (only approximately) orthogonal. The merocyanine form closest to this point should be the TCC form, but no local minimum could be obtained in the vicinity of this corner of the parameter space. From the schematic structure of the TCC form shown in Fig. 2 it is obvious that in this form strong steric hindrance exists between the oxygen atom and the two geminal methyl groups. Interestingly, no stable merocyanine form could be found for which $B=0^{\circ}$, i.e. for which the formal single bond in the merocyanine chain has a cisoid configuration. All attempts to optimize the corresponding structure finally lead to the corresponding transoid form. The only exception is the CCT form for which a shallow minimum could be found. In this isomer $B=67^{\circ}$, $i . e$. the formal "cis" bond is aiready strongly twisted. 
$\sqrt{\alpha} \sum_{\gamma}^{\beta}=0$
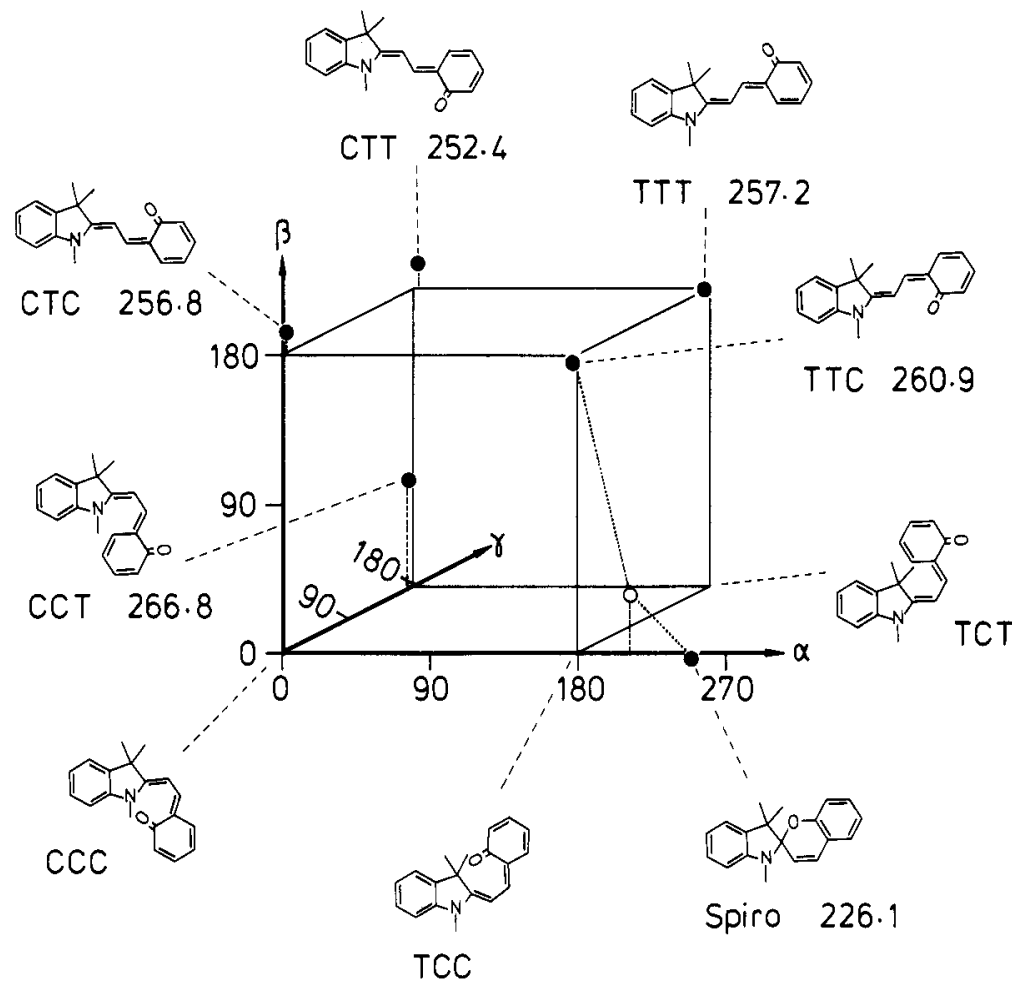

Fig. 2. Structural formulas of the spiro form and the 8 possible cis/trans isomers of the merocyanine form of BIPS. (The isomers are drawn only in their quinoidal mesomeric form.) Within the parameter space of the three dieder angles $\alpha, \quad B$, and $\gamma$, the merocyanine isomers occupy the 8 corners of a cube. The local energy minima found with the AM1 method are indicated by full circles. Numbers give the calculated enthalpy of formation in $\mathrm{kJ} / \mathrm{mole}$. The open circle represents the saddle point between the spiro form and the TTC isomer.

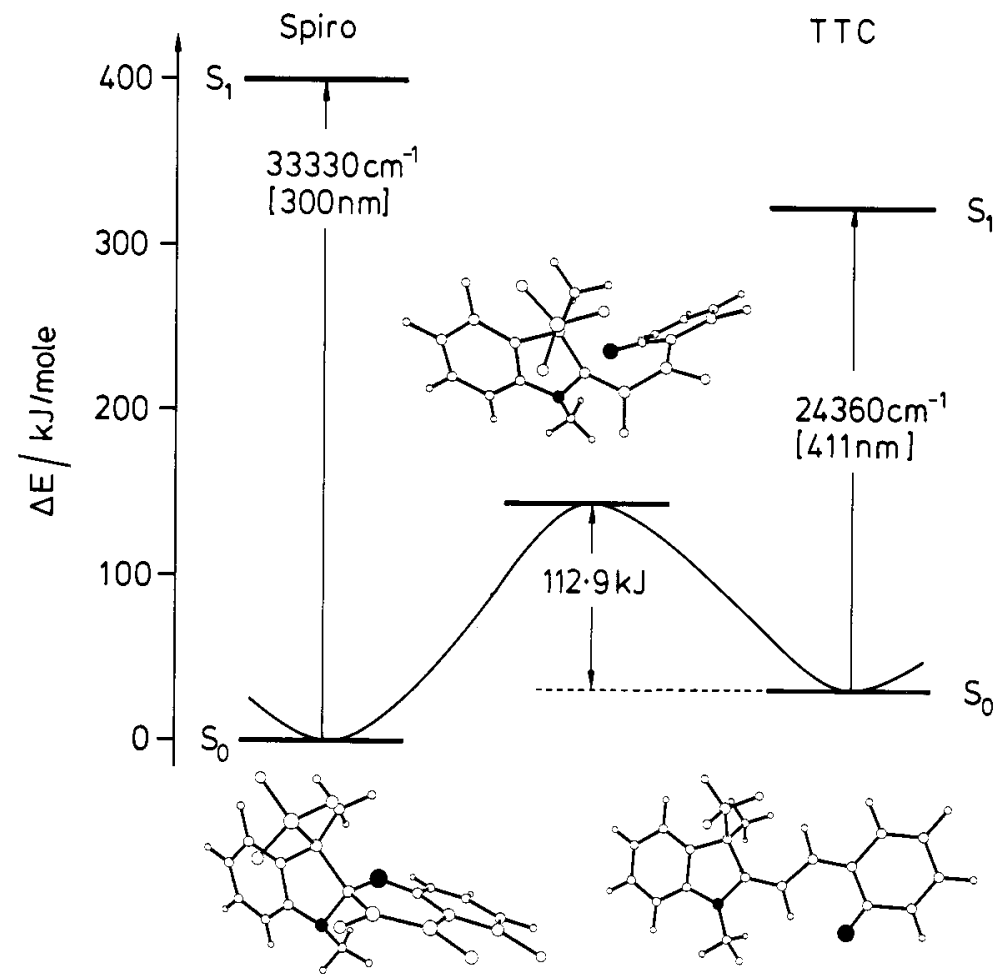

Fig. 3. Energy level diagram for the thermal reaction between the spiro form and the TTC merocyanine isomer of BIPS obtained with the AMl method. The optimized (on the SCF level) geometries of the two stable ground states and the transition state are also shown. 
Thus the stable merocyanine isomer closest to the spiro form of BIPS is found to be the TTC form. The formation of this isomer involves a simultaneous rotation of the angles $\alpha$ and $B$ by $73^{\circ}$ and $175^{\circ}$, respectively. The saddle point between these two forms on the ground state potential hypersurface is indicated by the open circle in Fig. 2.

The energy level diagram relevant to the discussion of the thermal reaction between the spiro form and the TTC isomer is shown in Fig. 3. The energies are given relative to that of the spiro ground state. The correction of the configuration interaction calculation has been included since these calculations also yield values for the vertical excitation energies. The three ball-and-stick models are the optimized geometries of the two ground states and the transition state. An activation energy of $112.9 \mathrm{~kJ} / \mathrm{mole}(26.9 \mathrm{kcal} / \mathrm{mole})$ is calculated for the thermal reaction from the merocyanine to the spiro form. For a solution in propanol, the experimental activation energy was found to be $71 \mathrm{~kJ} / \mathrm{mole}(17 \mathrm{kcal} / \mathrm{mole})(\mathrm{ref}$. 2). The agreement is quite reasonable in view of specific solvation effects (ref. 15, 16).

\section{TRANSIENT ABSORPTION SPECTRA WITH SUB-PS TIME RESOLUTION}

The pump-and-probe spectrometer for transient absorption is described in ref. 28 . An intense pump pulse at $308 \mathrm{~nm}$ initiates the photochemical reaction; it has a duration of 0.26 ps. After a variable delay, the absorption spectrum of the sample is recorded using a white-light continuum of 0.1 ps duration.

Figure 4 shows typical transient absorption spectra of BIPS and naphtho-BIPS in $n$-pentane, after some tens of ps.

Also shown as dashed lines are the spectra (fluorescence excitation) of the corresponding merocyanine form, generated by irradiation of the spiropyran in an argon matrix at $10 \mathrm{~K}$. The isomer spectra at low temperature are structured, and they must be assigned to the isomer labelled $B_{1}$ in (ref. 2, 3).
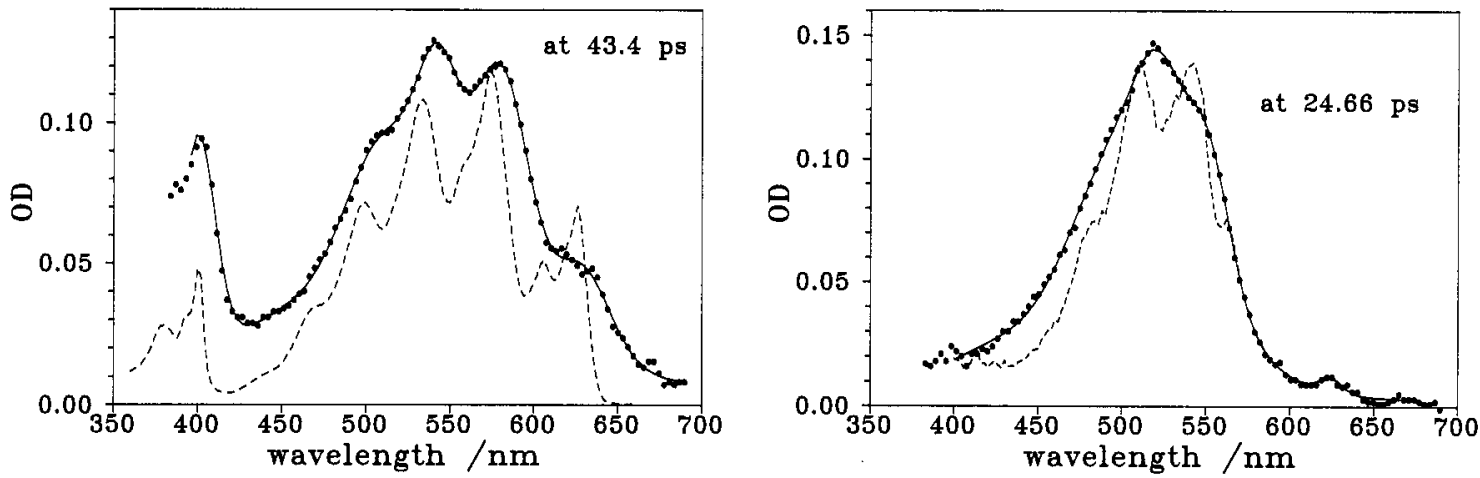

Fig. 4. Transient absorption spectra (dots), fits by Gaussian functions representing vibronic bands (solid lines), and fluorescence excitation spectra of merocyanine photoproducts in an argon matrix at $10 \mathrm{~K}$ (dashed 1 ines). Left: BIPS; right: naphtho-BIPS; both after UV excitation.

In the following, and for simplicity, let us consider the photochemistry of BIPS only. The spectral structure for the transient absorption spectrum from BIPS corresponds quite well with that of the $B_{1}$ isomer. In fact, the transient spectrum may be fitted with Gaussian functions (on an energy scale) representing the vibronic bands which are clearly discernible in the low-temperature spectrum. Comparison of the fits gives the spectral shift due to differences in solvation, and spectral broadening due to the relatively high internal temperature of the transient species.

Figures 5 and 6 give an overview of the spectral development up to $8 \mathrm{ps}$. Because of the low quantum yield for photocoloration of BIPS (ca. 6\%, ref. 2), intense pump pulses had to be used. This introduces transient absorption coincident with the pump pulse, in the range $\lambda \leqslant$ $470 \mathrm{~nm}$, due to multiphoton absorption by the solvent. A remnant of this artefact can also be seen in the transient absorption of naphtho-BIPS at earliest time. The spectral development for BIPS shows incipient spectral structure already at 2.1 ps. Cooling is indicated by slow steepening of the red absorption edge over tens of ps.

Kinetic traces of the development of merocyanine absorbance at the absorption maximum are shown in Fig. 7. Single exponential rise is given for comparison, with risetimes of 0.9 ps for BIPS and $1.4 \mathrm{ps}$ for naphtho-BIPS. Deviations at early time are significant, and they must be attributed to an ultrafast transient in the spiropyran - merocyanine conversion. 

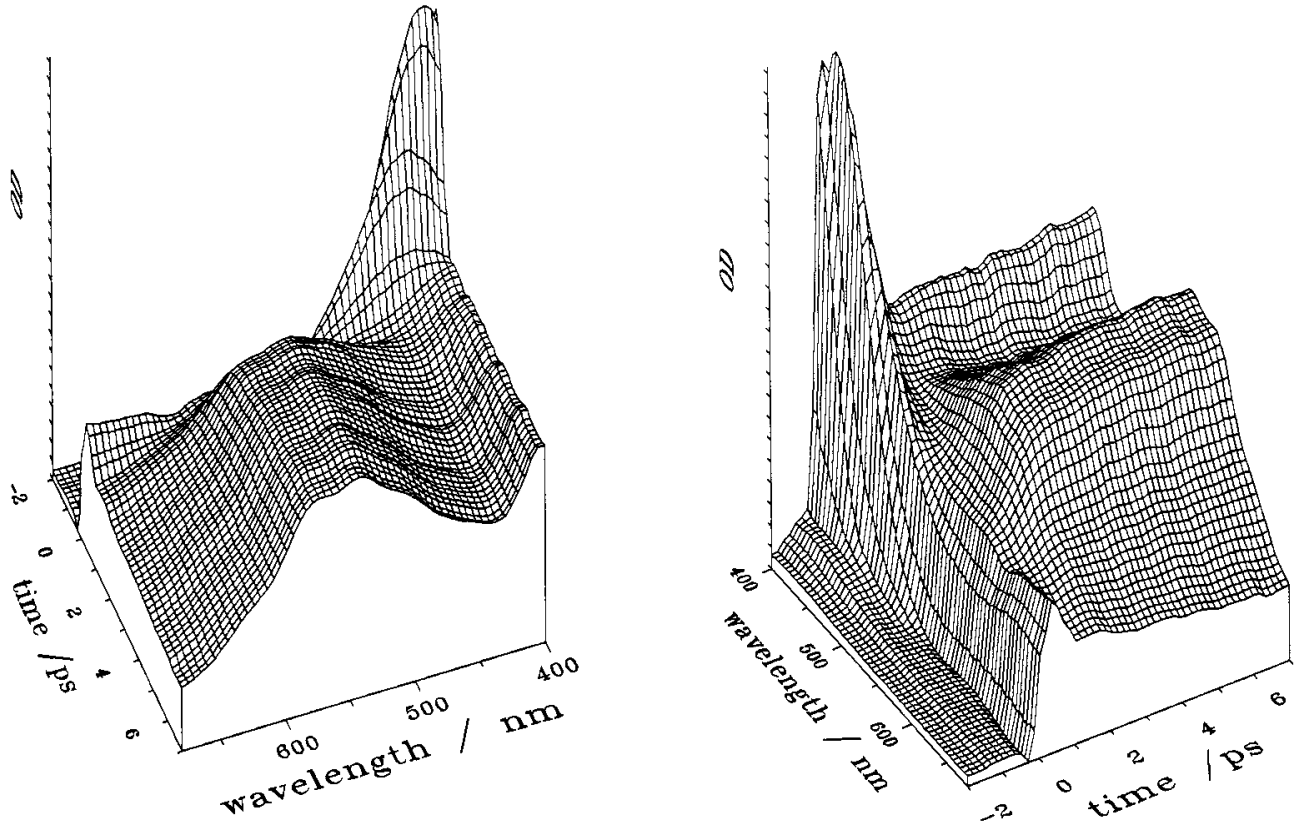

Fig. 5. Transient absorption of BIPS in $n$-pentane.
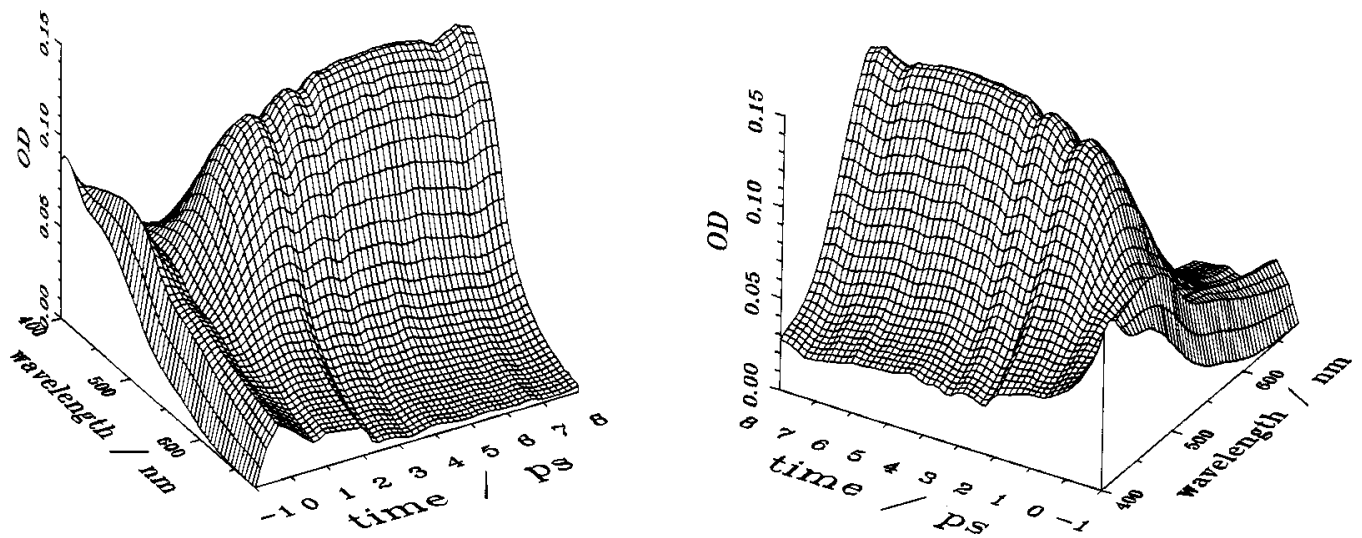

Fig. 6. Transient absorption of naphtho-BIPS in $n$-pentane.
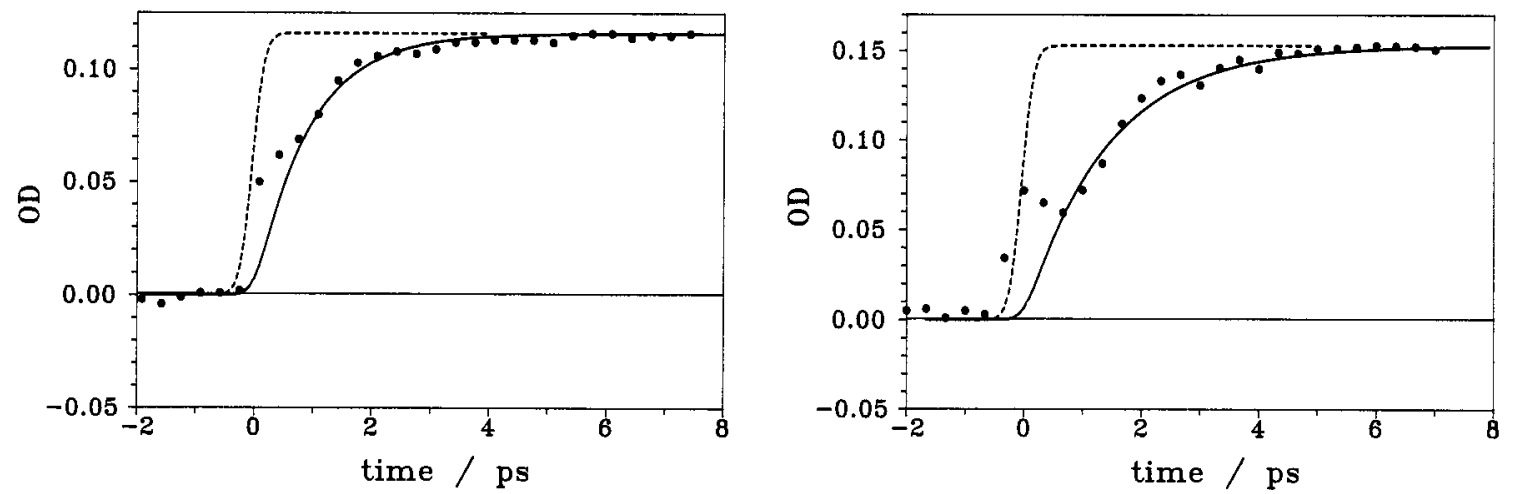

Fig. 7. Kinetic traces for the development of merocyanine absorbance, for solutions of BIPS (left) and naphtho-BIPS (right) in $n$-pentane. 


\section{DISCUSSION}

Let us return to the scheme in Fig. 2. A previous theoretical study of the ring opening reaction in chromene (ref. 29) had predicted that the photochemical, forward reaction involves a change in $\gamma$. We believe that the reaction path on the $S_{1}$ potential energy surface likely proceeds from the spiro form in the direction of the CCT isomer for steric reasons. The photochemical reaction proceeds even at $10 \mathrm{~K}$ in an argon matrix. Therefore the Sl surface can only have a very small - if any - activation barrier, and its slope along the path must be small even at a significant distance from the spiro form. Then the following picture would be consistent with our observations. The path skirts the saddle point of the ground state surface, and internal conversion occurs. A large fraction returns to ground state spiropyran. This explains a low quantum yield of photochemistry (ref. 4) and luminescence even at low temperature (ref. 8). The remainder unfolds to the TTC conformation with a time constant of 0.9 or 1.4 ps. Calculations predict that this isomer should rearrange in its $S_{1}$ excited state (ref. 30); it should therefore have a low quantum yield for fluorescence. This is also consistent with observations (ref. 3). In this context, the broad, ultrafast transient absorption coincident with the pump pulse must be assigned to excited state absorption from the unfolding spiropyran.

In conclusion: we find no evidence for a reasonably stable merocyanine isomer $X$ with a cisoid geometry. Instead, the primary photochemical reaction step of the unsubstituted spiropyran BIPS and similar spiropyrans should lead directly to a TTC isomer within 2 ps at room temperature.

\section{REFERENCES}

1. E. Fischer and Y. Hirshberg, J. Chem. Soc. 4522-4524 (1952).

2. T. Bercovici, R. Heiligman-Rim and E. Fischer, Mol. Photochem. 1, 23-55 (1969).

3. C. Balny, P. Douzou, T. Bercovici and E. Fischer, Mol. Photochem. 1, 225-233 (1969)

4. R.C. Bertelson. in: Photochromism, G.H. Brown (Ed.), Wiley-Interscience, New York (1971), p. 45 .

5. N.Y.C. Chu, Can. J. Chem. 61, 300-305 (1983).

6. S.M. Aldoshin, L.0. Atovmyan and O.A. Kozina, Bul1. Acad. Science USSR, Ser. Chem. 36, 169-172 (1987).

7. N.W. Tyer, Jr., and R.S. Becker, J.Amer. Chem. Soc. 92, 1289-1294 (1970).

8. N.W. Tyer, Jr., and R.S. Becker, J. Amer. Chem. Soc. g2, 1295-1302 (1970).

9. I.L. Belaits, T.D. Platonova and V.A. Barachevskii, Opt. Spectrosc. (USSR) 49 , 615-618 (1980)

10. M. Gehrtz, Chr. Bräuchle and J. Voitländer, J. Amer. Chem. Soc. 104, 2094-2101 (1982).

11. A.S. Kholmansky and K.M. Dumaev, J. Photochem. 31, 307-314 (1985).

12. K. Horie, K. Hirao, N. Kenmochi and I. Mita, Makromol. Chem., Rapid Commun. 9 , $267-273$ (1988).

13. H. Takahashi, K. Yoda, H. Isaka, T. Ohzeki and Y. Sakaino, Chem. Phys. Lett. 140, 90-94 (1987).

14. H. Takahashi, H. Murakawa, Y. Sakaino, T. Ohzeki, J. Abe and 0. Yamada, J. Photochem. A 45, 233-241 (1988).

15. K. Yoda, T. Ohzeki, T. Yuzawa and H. Takahashi, Spectrochim. Acta 45A, 855-862 (1989).

16. S.M. Aldosh in and L.0. Atovmyan, Mol. Cryst. Lig. Cryst. 149, 251-290 (1987).

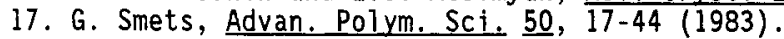

18. R. Richert, Chem. Phys. 122, 455-462 (1988).

19. K. Horje and I. Mita, Ädvan. Polym. Sci. 88, 79-128 (1989)

20. H. Eckhardt, A. Bose and V.A. Krongauz, Polymer 28, 1559-1564 (1987).

21. D.A. Reeves and F. Wilkinson, J. Chem. Soc., Faraday Trans. 2, 69, 1381-1390 (1973)

22. Y. Kalisky and D.J. Williams, Makromolecules 17, 292-296 (1984).

23. C. Lenoble and R.S. Becker, J. Phys. Chem. 90, 62-65 (1986).

24. C. Lenoble and R.S. Becker, J. Photochem. 34, 83-88 (1986).

25. R. Heiligman-Rim, Y. Hirshberg and E. Fischer, J. Phys. Chem. 66, 2465-2470, 2470-2477 (1962).

26. S.A. Krysanov and M.V. Alfimov, Laser Chem. 4, 129-138 (1984).

27. A. Kellmann, L. Lindqvist, S. Monti, F. Tfibel and R. Guglielmetti, J. Photochem. 28, 547-558 (1983)

28. N.P. Ernsting and M. Kaschke, to be published.

29. F. Zerbetto, S. Monti and G. Orlandi, J. Chem. Soc., Faraday Trans. 2, 80, $1513-$ 1527 (1984).

30. B. Dick, to be published. 